\title{
FIRST EXIT TIME OF A LÉVY FLIGHT FROM A BOUNDED REGION IN $\mathbb{R}^{N}$
}

\author{
YOORA KIM,* University of Ulsan \\ IREM KOPRULU $^{* *}$ AND \\ NESS B. SHROFF, ${ }^{* * *}$ The Ohio State University
}

\begin{abstract}
In this paper we characterize the mean and the distribution of the first exit time of a Lévy flight from a bounded region in $N$-dimensional spaces. We characterize tight upper and lower bounds on the tail distribution of the first exit time, and provide the exact asymptotics of the mean first exit time for a given range of step-length distribution parameters.
\end{abstract}

Keywords: Lévy flight; first exit time; first passage time; bounded region 2010 Mathematics Subject Classification: Primary 60J75

Secondary 60G50

\section{Model description and problem statement}

\subsection{Lévy flight}

A Lévy flight is a class of random walks that is characterized by a heavy-tailed step-length distribution. Let $\left\{Y_{n}\right\}_{n \in \mathbb{N}}$ be a sequence of random vectors that denote the position of an object that moves according to a Lévy flight process in $\mathbb{R}^{N}, N \geq 1$. We assume that the random walk starts at the origin. Then, for the one-dimensional case, $Y_{n}$ can be written as

$$
Y_{n}=\sum_{k=1}^{n} X_{k} \in \mathbb{R} .
$$

In (1.1), $X_{k}$ denotes the $k$ th signed step-length, and is independent and identically distributed (i.i.d.) across $k$ according to an $\alpha$-stable distribution. Let $X$ be the generic random variable for $X_{k}$. Then the characteristic function of $X$, defined as $\varphi_{X}(t):=\mathbb{E}\left[\mathrm{e}^{\mathrm{i} t X}\right]$, is determined by the parameter tuple $(\alpha, \beta, \gamma, \delta)$ as

$$
\varphi_{X}(t)= \begin{cases}\exp \left(-\gamma^{\alpha}|t|^{\alpha}\left[1-\mathrm{i} \beta\left(\tan \frac{\pi \alpha}{2}\right)(\operatorname{sgn} t)\right]+\mathrm{i} \delta t\right), & \alpha \neq 1, \\ \exp \left(-\gamma|t|\left[1+\mathrm{i} \beta \frac{2}{\pi}(\operatorname{sgn} t) \log |t|\right]+\mathrm{i} \delta t\right), & \alpha=1 .\end{cases}
$$

In (1.2), the stability index $\alpha \in(0,2]$ and the skewness parameter $\beta \in[-1,1]$ determine the form of the distribution in terms of the tail asymptotics and the skewness, and $\gamma>0$ and $\delta \in \mathbb{R}$ are parameters for the scale and the shift, respectively [13].

\footnotetext{
Received 1 October 2013; revision received 1 July 2014.

* Postal address: Department of Mathematics, University of Ulsan, 93 Daehak-ro, Nam-gu, Ulsan, South Korea. Email address: yrkim@ulsan.ac.kr

** Postal address: Department of Electrical and Computer Engineering, The Ohio State University, Columbus, $\mathrm{OH}$ 43210, USA. Email address: irem.koprulu@gmail.com

*** Postal address: Departments of Electrical and Computer Engineering and Computer Science and Engineering, The Ohio State University, Columbus, OH 43210, USA. Email address: shroff.11@ osu.edu
} 
For the $N$-dimensional $(N \geq 2)$ case, $Y_{n}$ can be written using hyperspherical coordinates as

$$
Y_{n}=\sum_{k=1}^{n}\left|X_{k}\right| \Lambda\left(\theta_{k}\right) \in \mathbb{R}^{N}
$$

In (1.3), $\left|X_{k}\right|$ denotes the absolute value of the $k$ th signed step-length $X_{k}$ and corresponds to the radial coordinate. Then $\theta_{k}=\left(\theta_{k, m}\right)_{m=1, \ldots, N-1}$ is an $(N-1)$-tuple of angular coordinates, and $\Lambda\left(\theta_{k}\right)$ denotes the unit-length direction vector associated with it. In our model, the angular coordinates $\left(\theta_{k, m}\right)_{m=1, \ldots, N-1}$ are chosen independently of the step-length and each other, and uniformly at random from the interval $[0, \pi)$ for $m=1, \ldots, N-2$, and $[0,2 \pi)$ for $m=N-1$, which results in an isotropic random walk. The unit-length direction vector $\Lambda(\cdot)$ is defined for $\boldsymbol{\omega}=\left(\omega_{1}, \ldots, \omega_{N-1}\right) \in \Omega:=[0, \pi)^{N-2} \times[0,2 \pi)$ by $\Lambda(\boldsymbol{\omega})=\left(\Lambda_{1}(\boldsymbol{\omega}), \ldots, \Lambda_{N}(\boldsymbol{\omega})\right)$, where

$$
\begin{aligned}
\Lambda_{1}(\boldsymbol{\omega}) & =\cos \left(\omega_{1}\right), \\
\Lambda_{2}(\boldsymbol{\omega}) & =\sin \left(\omega_{1}\right) \cos \left(\omega_{2}\right), \\
\Lambda_{3}(\boldsymbol{\omega}) & =\sin \left(\omega_{1}\right) \sin \left(\omega_{2}\right) \cos \left(\omega_{3}\right), \\
& \vdots \\
\Lambda_{N-1}(\boldsymbol{\omega}) & =\sin \left(\omega_{1}\right) \ldots \sin \left(\omega_{N-2}\right) \cos \left(\omega_{N-1}\right), \\
\Lambda_{N}(\boldsymbol{\omega}) & =\sin \left(\omega_{1}\right) \ldots \sin \left(\omega_{N-2}\right) \sin \left(\omega_{N-1}\right) .
\end{aligned}
$$

In this paper we consider $\alpha$-stable distributions with $\beta=\delta=0$ in which case the characteristic function reduces to $\varphi_{X}(t)=\mathrm{e}^{-\gamma^{\alpha}|t|^{\alpha}}$. This results in a symmetric distribution for the signed step-length and a symmetric Lévy flight in $\mathbb{R}$. Furthermore, we focus on $\alpha \in(0,2)$, since for $\alpha=2$ the resulting $\alpha$-stable distribution is Gaussian and no longer heavy-tailed (i.e. the resulting random walk is not a Lévy flight). For this set of parameters, the tail of the $\alpha$-stable distribution asymptotically behaves as

$$
\mathbb{P}(X>x)=\mathbb{P}(X<-x) \sim \frac{\Gamma(\alpha)}{\pi} \sin \left(\frac{\pi \alpha}{2}\right) \gamma^{\alpha} x^{-\alpha} .
$$

Here, $\Gamma(\cdot)$ is the gamma function, and for any two functions $g, h: \mathbb{R} \rightarrow \mathbb{R}$, we write $g(x) \sim$ $h(x)$ to denote $\lim _{x \rightarrow \infty} g(x) / h(x)=1$. Throughout this paper, we adopt the following notation to indicate asymptotic behavior:

$$
\begin{array}{ll}
g(x)=O(h(x)) & \text { if } \limsup _{x \rightarrow \infty} \frac{g(x)}{h(x)}<\infty, \\
g(x)=\Omega(h(x)) & \text { if } h(x)=O(g(x)), \\
g(x)=\Theta(h(x)) & \text { if } g(x)=O(h(x)), \quad \text { and } \quad g(x)=\Omega(h(x)) .
\end{array}
$$

\subsection{First exit time from a bounded region}

The quantity of interest in this paper is the first exit time of a Lévy flight from a bounded region, in particular a closed $N$-ball of radius $R$. We provide the formal definition of the first exit time in Definition 1.1.

Definition 1.1. For a given $R>0$, the first exit time $\tau_{R}$ is defined as

$$
\tau_{R}:=\inf \left\{n \in \mathbb{N}:\left|Y_{n}\right|>R\right\},
$$

where $\left|Y_{n}\right|$ denotes the Euclidean norm of $Y_{n}$. 
Since $X_{k}$ is not identically 0 for any $k \in \mathbb{N}, \tau_{R}$ is a proper random variable, i.e.

$$
\lim _{t \rightarrow \infty} \mathbb{P}\left(\tau_{R}>t\right)=0 .
$$

In this paper we focus on characterizing the behavior of the distribution and the mean of $\tau_{R}$ as a function of $R$.

Note that the first exit time is sometimes also referred to as the first passage time in the literature. In our work we distinguish between them as follows. The first exit time is concerned with a bounded region, and the first passage time is concerned with a semi-infinite interval in one-dimension. We define the first passage time as $v=v_{x}:=\inf \left\{n \in \mathbb{N}: Y_{n}>x\right\}$. A wellestablished result on the first passage time is the Sparre Andersen theorem which states that for any discrete-time random walk with a continuous and symmetric step-length distribution, the first passage time density $f_{v}(t)$ scales asymptotically as $t^{-3 / 2}$ [1]. In this paper, we use $F_{W}(w):=\mathbb{P}(W \leq w)$ to denote the cumulative distribution function (CDF) of a random variable $W$. If it exists we denote the probability density function (PDF) of $W$ by $f_{W}(w)$.

Pruitt [9] derived upper and lower bounds on the distribution and the mean of the first exit time for any random walk in $\mathbb{R}^{N}$. For one-dimensional Lévy flights, the average first exit time from a bounded interval has been studied by Buldyrev et al. [2], [3]. A later study by Dybiec et al. [5], however, pointed out that non-local boundary conditions have to be considered due to the heavy-tailed step-lengths of a Lévy flight, and hence the analytical results in [2], [3] are correct only for $\alpha=2$. Dybiec et al. [5] provided a numerical study to verify their results, and the analytical solution is left open. For two-dimensional Lévy flights, there is a very recent study by Vahabi et al. [10], who provided numerical results on the mean first exit time. For general $N$-dimensional Lévy flights, there are no analytical results for either the distribution or moments of the first exit time from a bounded region.

An analytical solution for the distribution and the mean of the first exit time of a Lévy flight in $\mathbb{R}^{N}$ is known for the diffusion limit, i.e. $\alpha$-stable Lévy motion [11]. In one-dimensional space, Katzav and Adda-Bedia [8] and Zoia et al. [12] derived the distribution and the moments of the first exit time. In general $N$-dimensional spaces, Getoor [7] established exact expressions for the first and second moments of the first exit time. Recently, Chen et al. [4] derived two-sided estimates for the heat kernel of a Dirichlet fractional Laplacian in $\mathbb{R}^{N}$. From [4, Theorem 1.1(ii)] we can deduce that the tail distribution of the first exit time from an $N$-ball has exponential upper and lower bounds.

In this paper we analyze the distribution and the mean of the first exit time of Lévy flights in $\mathbb{R}^{N}$. Our approach is to formulate the tail distribution of the first exit time in a recursive manner as $\mathbb{P}\left(\tau_{R}>n+1\right)=a_{n} \mathbb{P}\left(\tau_{R}>n\right)$ and derive bounds on the factor $a_{n}$ uniformly over all $n \in \mathbb{N}$. Based on this approach, we prove that the tail distribution is bounded above and below by exponential functions. The efficacy of our bounds is illustrated by the fact that they translate into an order-wise tight bound on the mean first exit time for the stability index $\alpha \in(0,1)$.

\section{Analytic results on the first exit time}

In this section we state and prove our main results on the first exit time $\tau_{R}$ of a Lévy flight from an $N$-ball of radius $R$. We begin by providing upper and lower bounds on the tail probability of $\tau_{R}$ in Theorem 2.1. As a consequence of Theorem 2.1, we next analyze the asymptotics of the mean first exit time $\mathbb{E}\left[\tau_{R}\right]$ in Corollary 2.1 as the radius $R$ increases. Finally, we establish the dependence of the first exit time on the scale parameter $\gamma$ of the $\alpha$-stable distribution in Proposition 2.1. 
Theorem 2.1. For a Lévy flight in $\mathbb{R}^{N}$, the first exit time from a ball of radius $R$ is exponentially bounded, i.e. there exists $0<l_{R} \leq u_{R}<1$ such that

$$
\left(l_{R}\right)^{n} \leq \mathbb{P}\left(\tau_{R}>n\right) \leq\left(u_{R}\right)^{n} \text { for all } n \in \mathbb{N},
$$

where $l_{R}$ and $u_{R}$ are given as

$$
l_{R}=\frac{1}{z_{R}} \int_{0}^{z_{R}} F_{|X|}(x) \mathrm{d} x, \quad u_{R}=F_{|X|}(R),
$$

where $z_{R}:=2 R$ for $N=1$ and $z_{R}:=R$ for $N \geq 2$.

Remark 2.1. In [9], Pruitt derived bounds on the distribution of the extreme value $M_{n}=$ $\max _{i \leq n}\left|S_{i}\right|$ for any random walk $\left\{S_{n}\right\}_{n \in \mathbb{N}}$ in $\mathbb{R}^{N}$. When we apply [9] to a Lévy flight, we obtain the following bounds:

$$
1-v_{R} n \leq \mathbb{P}\left(\tau_{R}>n\right) \leq \frac{\zeta_{R}}{n} \quad \text { for all } n \in \mathbb{N}
$$

where $v_{R}$ and $\zeta_{R}$ are constants that scale as $\Theta\left(R^{-\alpha}\right)$ and $\Theta\left(R^{\alpha}\right)$, respectively. Since our bounds in Theorem 2.1 are exponential functions of $n$ whereas the bounds in (2.3) are polynomial functions, there exists $\hat{n} \in \mathbb{N}$ such that

$$
1-v_{R} n \leq\left(l_{R}\right)^{n} \leq \mathbb{P}\left(\tau_{R}>n\right) \leq\left(u_{R}\right)^{n} \leq \frac{\zeta_{R}}{n} \text { for all } n \geq \hat{n} .
$$

That is, after a certain point $n \geq \hat{n}$, our bounds in Theorem 2.1 become tighter than the bounds obtained by applying Pruitt's result to a Lévy flight.

An analogous result to Theorem 2.1 can be obtained for an $\alpha$-stable Lévy motion in $\mathbb{R}^{N}$. From [4, Theorem 1.1(ii)], we can deduce that the tail distribution of the first exit time from an $N$-ball has exponential upper and lower bounds as

$$
\eta_{1} \mathrm{e}^{-\lambda t} \leq \mathbb{P}\left(\tau_{R}>t\right) \leq \eta_{2} \mathrm{e}^{-\lambda t}
$$

where $\lambda$ is the smallest eigenvalue of the Dirichlet fractional Laplacian, and $\eta_{1}$ and $\eta_{2}$ are positive constants.

To prove Theorem 2.1, we need three lemmas. Lemma 2.1 is concerned with the distribution of the absolute value of an $\alpha$-stable distributed random variable $X$, and is a direct consequence of the bell-shaped nature of $\alpha$-stable distributions [6]. The proof of Lemma 2.1 is given in Appendix A.

Lemma 2.1. The $C D F F_{|X|}(\cdot)$ is infinitely differentiable on its domain $[0, \infty)$. Hence, the PDF of $|X|$ exists. Moreover, the PDF is monotone and satisfies $f_{|X|}\left(x_{l}\right) \geq f_{|X|}\left(x_{u}\right)$ for $0 \leq x_{l} \leq x_{u}$.

In Lemma 2.2 we explore the distribution of the $n$th position of a Lévy flight $\left\{Y_{n}\right\}_{n \in \mathbb{N}}$ conditioned on the event that all positions up to time $n$ lie inside the ball of radius $R$. In particular, we examine the existence, the continuity, and the monotonicity of the conditional density function of $\left|Y_{n}\right|$. The proof of Lemma 2.2 is given in Appendix B.

Lemma 2.2. The conditional density function $f_{\left|Y_{n}\right|}\left(y \mid \tau_{R}>n\right)$ exists for all $n \in \mathbb{N}$, and is continuous over its domain $[0, R]$. In addition, for $0 \leq y_{l} \leq y_{u} \leq R$, we have

$$
f_{\left|Y_{n}\right|}\left(y_{l} \mid \tau_{R}>n\right) \geq f_{\left|Y_{n}\right|}\left(y_{u} \mid \tau_{R}>n\right) .
$$


In Lemma 2.3 we investigate the probability of exiting the ball of radius $R$ at the next step as a function of the current position of the Lévy flight. We show that the Lévy flight is less likely to exit the ball when its current position is closer to the center of the ball. The proof of Lemma 2.3 is given in Appendix C.

Lemma 2.3. Let $G:[0, R] \rightarrow[0,1]$ be defined as $G(y):=\mathbb{P}\left(\left|Y_{n+1}\right| \leq R|| Y_{n} \mid=y\right)$. Then, for the one-dimensional Lévy flight, we have

$$
G(y)=\frac{1}{2} F_{|X|}(R-y)+\frac{1}{2} F_{|X|}(R+y),
$$

and for the $N$-dimensional $(N \geq 2)$ Lévy flight, we have

$$
G(y) \geq F_{|X|}(R-y) .
$$

Regardless of the dimension, for any Lévy flight, we have for $0 \leq y_{l} \leq y_{u} \leq R$,

$$
G\left(y_{l}\right) \geq G\left(y_{u}\right) .
$$

Proof of Theorem 2.1. We complete the proof in two steps. First, we show that for any $n \in \mathbb{N}$, the distribution $\mathbb{P}\left(\tau_{R}>n\right)$ can be written recursively with the initial value $\mathbb{P}\left(\tau_{R}>1\right)=\mathbb{P}\left(\left|Y_{1}\right| \leq R\right)=F_{|X|}(R)$ as

$$
\mathbb{P}\left(\tau_{R}>n+1\right)=\mathbb{P}\left(\tau_{R}>n\right) \int_{0}^{R} G(y) f_{\left|Y_{n}\right|}\left(y \mid \tau_{R}>n\right) \mathrm{d} y,
$$

where $G(\cdot)$ is defined in Lemma 2.3, and the existence of $f_{\left|Y_{n}\right|}\left(\cdot \mid \tau_{R}>n\right)$ has already been shown in Lemma 2.2. Second, we show that the integral in $(2.8)$ is bounded by

$$
l_{R} \leq \int_{0}^{R} G(y) f_{\left|Y_{n}\right|}\left(y \mid \tau_{R}>n\right) \mathrm{d} y \leq u_{R} \quad \text { for all } n \in \mathbb{N} .
$$

Combining (2.8) and (2.9) yields Theorem 2.1.

Note that $\left\{\tau_{R}>n\right\}=\left\{\left|Y_{k}\right| \leq R, k=1,2, \ldots, n\right\}$. Hence, by conditioning on the event $\left\{\tau_{R}>n\right\}$ we can rewrite the probability $\mathbb{P}\left(\tau_{R}>n+1\right)$ as

$$
\begin{aligned}
\mathbb{P}\left(\tau_{R}>n+1\right) & =\mathbb{P}\left(\tau_{R}>n+1 \mid \tau_{R}>n\right) \mathbb{P}\left(\tau_{R}>n\right) \\
& =\mathbb{P}\left(\left|Y_{k}\right| \leq R, k=1,2, \ldots, n+1|| Y_{k} \mid \leq R, k=1,2, \ldots, n\right) \mathbb{P}\left(\tau_{R}>n\right) \\
& =\mathbb{P}\left(\left|Y_{n+1}\right| \leq R \mid \tau_{R}>n\right) \mathbb{P}\left(\tau_{R}>n\right) .
\end{aligned}
$$

We focus on the probability $\mathbb{P}\left(\left|Y_{n+1}\right| \leq R \mid \tau_{R}>n\right)$ in (2.10) for the $N$-dimensional $(N \geq 2)$ case, and omit the analysis for the one-dimensional case. For $y \in[0, R]$ and $\omega \in \Omega$, the joint conditional density function $f_{\left|Y_{n}\right|, L\left(Y_{n}\right)}\left(y, \omega \mid \tau_{R}>n\right)$ exists by Lemma 2.2, and given the isotropic nature of the Lévy flight, it can be written as

$$
f_{\left|Y_{n}\right|, L\left(Y_{n}\right)}\left(y, \omega \mid \tau_{R}>n\right)=\frac{1}{2 \pi^{N-1}} f_{\left|Y_{n}\right|}\left(y \mid \tau_{R}>n\right) .
$$

Thus, we can write $\mathbb{P}\left(\left|Y_{n+1}\right| \leq R \mid \tau_{R}>n\right)$ by conditioning on $Y_{n}$ as

$$
\begin{aligned}
& \mathbb{P}\left(\left|Y_{n+1}\right| \leq R \mid \tau_{R}>n\right) \\
& \quad=\int_{0}^{R} \int_{\Omega} \mathbb{P}\left(\left|Y_{n+1}\right| \leq R \mid Y_{n}=y \Lambda(\omega), \tau_{R}>n\right) f_{\left|Y_{n}\right|, L\left(Y_{n}\right)}\left(y, \omega \mid \tau_{R}>n\right) \mathrm{d} \omega \mathrm{d} y .
\end{aligned}
$$


The probability inside the integral in (2.12) reduces to

$$
\begin{aligned}
\mathbb{P}\left(\left|Y_{n+1}\right| \leq R \mid Y_{n}=y \Lambda(\omega), \tau_{R}>n\right) & =\mathbb{P}\left(\left|Y_{n+1}\right| \leq R \mid Y_{n}=y \Lambda(\omega)\right) \\
& =\mathbb{P}\left(\left|Y_{n+1}\right| \leq R|| Y_{n} \mid=y\right)
\end{aligned}
$$

where the first and the second equalities follow from the Markov property and the isotropy of the Lévy flight, respectively. Substituting (2.11) and (2.13) into (2.12), we have

$$
\begin{aligned}
& \mathbb{P}\left(\left|Y_{n+1}\right| \leq R \mid \tau_{R}>n\right) \\
& \quad=\int_{0}^{R} \int_{\Omega} \mathbb{P}\left(\left|Y_{n+1}\right| \leq R|| Y_{n} \mid=y\right) \frac{1}{2 \pi^{N-1}} f_{\left|Y_{n}\right|}\left(y \mid \tau_{R}>n\right) \mathrm{d} \omega \mathrm{d} y \\
& \quad=\int_{0}^{R} \mathbb{P}\left(\left|Y_{n+1}\right| \leq R|| Y_{n} \mid=y\right) f_{\left|Y_{n}\right|}\left(y \mid \tau_{R}>n\right) \mathrm{d} y \\
& \quad=\int_{0}^{R} G(y) f_{\left|Y_{n}\right|}\left(y \mid \tau_{R}>n\right) \mathrm{d} y .
\end{aligned}
$$

Thus, combining (2.10) and (2.14), we obtain the recursion formula (2.8).

We will next prove the upper bound in (2.9). From (2.7) in Lemma 2.3, we have

$$
\int_{0}^{R} G(y) f_{\left|Y_{n}\right|}\left(y \mid \tau_{R}>n\right) \mathrm{d} y \leq G(0) \int_{0}^{R} f_{\left|Y_{n}\right|}\left(y \mid \tau_{R}>n\right) \mathrm{d} y=G(0),
$$

and $G(0):=\mathbb{P}\left(\left|Y_{n+1}\right| \leq R|| Y_{n} \mid=0\right)=\mathbb{P}\left(\left|X_{n+1}\right| \leq R\right)=F_{|X|}(R)$. This gives the upper bound $u_{R}=F_{|X|}(R)$ in (2.9).

Finally, we prove the lower bound in (2.9). By (2.4) in Lemma 2.2, we have

$$
R f_{\left|Y_{n}\right|}\left(R \mid \tau_{R}>n\right) \leq \int_{0}^{R} f_{\left|Y_{n}\right|}\left(y \mid \tau_{R}>n\right) \mathrm{d} y \leq R f_{\left|Y_{n}\right|}\left(0 \mid \tau_{R}>n\right) .
$$

Since $\int_{0}^{R} f_{\left|Y_{n}\right|}\left(y \mid \tau_{R}>n\right) \mathrm{d} y=1$, we have

$$
f_{\left|Y_{n}\right|}\left(R \mid \tau_{R}>n\right) \leq \frac{1}{R} \leq f_{\left|Y_{n}\right|}\left(0 \mid \tau_{R}>n\right)
$$

In addition, since $f_{\left|Y_{n}\right|}\left(\cdot \mid \tau_{R}>n\right)$ is a continuous function (by Lemma 2.2), we can apply the intermediate value theorem to show the existence of $\hat{y}=\hat{y}_{n} \in[0, R]$ such that $f_{\left|Y_{n}\right|}\left(\hat{y} \mid \tau_{R}>\right.$ $n)=1 / R$. In the following, we will show that the integral in (2.9) is bounded below by $1 / R \int_{0}^{R} G(y) \mathrm{d} y$ by computing the difference over two separate intervals of integration $[0, \hat{y}]$ and $(\hat{y}, R]$ :

$$
\begin{aligned}
& \int_{0}^{R} G(y) f_{\left|Y_{n}\right|}\left(y \mid \tau_{R}>n\right) \mathrm{d} y-\frac{1}{R} \int_{0}^{R} G(y) \mathrm{d} y \\
& \quad=\int_{0}^{\hat{y}} G(y)\left(f_{\left|Y_{n}\right|}\left(y \mid \tau_{R}>n\right)-\frac{1}{R}\right) \mathrm{d} y+\int_{\hat{y}}^{R} G(y)\left(f_{\left|Y_{n}\right|}\left(y \mid \tau_{R}>n\right)-\frac{1}{R}\right) \mathrm{d} y .
\end{aligned}
$$


For $0 \leq y \leq \hat{y}$, we have $f_{\left|Y_{n}\right|}\left(y \mid \tau_{R}>n\right) \geq f_{\left|Y_{n}\right|}\left(\hat{y} \mid \tau_{R}>n\right)=1 / R$ by (2.4) in Lemma 2.2. Hence, the first integral in the right-hand side of (2.15) is bounded by

$$
\begin{aligned}
\int_{0}^{\hat{y}} G(y)\left(f_{\left|Y_{n}\right|}\left(y \mid \tau_{R}>n\right)-\frac{1}{R}\right) \mathrm{d} y & \geq \inf _{0 \leq y \leq \hat{y}}\{G(y)\} \int_{0}^{\hat{y}}\left(f_{\left|Y_{n}\right|}\left(y \mid \tau_{R}>n\right)-\frac{1}{R}\right) \mathrm{d} y \\
& =G(\hat{y}) \int_{0}^{y}\left(f_{\left|Y_{n}\right|}\left(y \mid \tau_{R}>n\right)-\frac{1}{R}\right) \mathrm{d} y,
\end{aligned}
$$

where we have used $\inf _{0 \leq y \leq \hat{y}}\{G(y)\}=G(\hat{y})$ by (2.7) in Lemma 2.3. Similarly, the second integral in the right-hand side of (2.15) is bounded by

$$
\begin{aligned}
\int_{\hat{y}}^{R} G(y)\left(f_{\left|Y_{n}\right|}\left(y \mid \tau_{R}>n\right)-\frac{1}{R}\right) \mathrm{d} y & \geq \sup _{\hat{y} \leq y \leq R}\{G(y)\} \int_{\hat{y}}^{R}\left(f_{\left|Y_{n}\right|}\left(y \mid \tau_{R}>n\right)-\frac{1}{R}\right) \mathrm{d} y \\
& =G(\hat{y}) \int_{\hat{y}}^{R}\left(f_{\left|Y_{n}\right|}\left(y \mid \tau_{R}>n\right)-\frac{1}{R}\right) \mathrm{d} y .
\end{aligned}
$$

Combining (2.15), (2.16), and (2.17), we have

$$
\begin{aligned}
\int_{0}^{R} G(y) f_{\left|Y_{n}\right|}\left(y \mid \tau_{R}>n\right) \mathrm{d} y-\frac{1}{R} \int_{0}^{R} G(y) \mathrm{d} y & \geq G(\hat{y}) \int_{0}^{R}\left(f_{\left|Y_{n}\right|}\left(y \mid \tau_{R}>n\right)-\frac{1}{R}\right) \mathrm{d} y \\
& =G(\hat{y})(1-1) \\
& =0 .
\end{aligned}
$$

That is, the integral in (2.8) is bounded below by

$$
\int_{0}^{R} G(y) f_{\left|Y_{n}\right|}\left(y \mid \tau_{R}>n\right) \mathrm{d} y \geq \frac{1}{R} \int_{0}^{R} G(y) \mathrm{d} y .
$$

Now we consider $1 / R \int_{0}^{R} G(y) \mathrm{d} y$ for the one-dimensional Lévy flight. By applying (2.5) in Lemma 2.3 and a change of variables, we have

$$
\begin{aligned}
\frac{1}{R} \int_{0}^{R} G(y) \mathrm{d} y & =\frac{1}{2 R} \int_{0}^{R} F_{|X|}(R-y) \mathrm{d} y+\frac{1}{2 R} \int_{0}^{R} F_{|X|}(R+y) \mathrm{d} y \\
& =\frac{1}{2 R} \int_{0}^{R} F_{|X|}(y) \mathrm{d} y+\frac{1}{2 R} \int_{R}^{2 R} F_{|X|}(y) \mathrm{d} y \\
& =\frac{1}{2 R} \int_{0}^{2 R} F_{|X|}(y) \mathrm{d} y \\
& =l_{R} .
\end{aligned}
$$

Next, we consider $1 / R \int_{0}^{R} G(y)$ dy for the $N$-dimensional $(N \geq 2)$ Lévy flight. Again, by applying (2.6) in Lemma 2.3 and a change of variables, we have

$$
\frac{1}{R} \int_{0}^{R} G(y) \mathrm{d} y \geq \frac{1}{R} \int_{0}^{R} F_{|X|}(R-y) \mathrm{d} y=\frac{1}{R} \int_{0}^{R} F_{|X|}(y) \mathrm{d} y=l_{R} .
$$

Combining (2.18), (2.19), and (2.20) yields the lower bound in (2.9). This completes the proof of Theorem 2.1. 
Using the result in Theorem 2.1, we can derive bounds on the mean first exit time $\mathbb{E}\left[\tau_{R}\right]$. In Corollary 2.1, we show that the bounds we provide are asymptotically tight as the radius $R$ increases for the stability index $\alpha \in(0,1)$.

Corollary 2.1. The mean first exit time of a Lévy flight in $\mathbb{R}^{N}$ is bounded by

$$
\frac{1}{1-l_{R}} \leq \mathbb{E}\left[\tau_{R}\right] \leq \frac{1}{1-u_{R}},
$$

where $l_{R}$ and $u_{R}$ are given in (2.2). From the bounds, the scaling behavior of $\mathbb{E}\left[\tau_{R}\right]$ with respect to $R$ is given as

$$
\mathbb{E}\left[\tau_{R}\right]=\Theta\left(R^{\alpha}\right) \text { for } \alpha \in(0,1) .
$$

Note that our bounds are tight in an order sense for a Lévy flight in $\mathbb{R}^{N}$ with $\alpha \in(0,1)$. For $\alpha=1$, we have $\mathbb{E}\left[\tau_{R}\right]=\Omega(R / \log (R))$ and $\mathbb{E}\left[\tau_{R}\right]=O(R)$, while for $\alpha \in(1,2)$ we have $\mathbb{E}\left[\tau_{R}\right]=\Omega(R)$ and $\mathbb{E}\left[\tau_{R}\right]=O\left(R^{\alpha}\right)$. In [9, Theorem 1], Pruitt establishes bounds on the mean first exit time for any random walk in $\mathbb{R}^{N}$ by using the bounds on the distribution of the extreme value $M_{n}$ that we mention in Remark 2.1. When we apply [9, Theorem 1] to a Lévy flight in $\mathbb{R}^{N}$, we obtain

$$
\mathbb{E}\left[\tau_{R}\right]=\Theta\left(R^{\alpha}\right) \quad \text { for } \alpha \in(0,2) .
$$

Comparing (2.21) with (2.22), we arrive at the same order result for a restricted range of $\alpha \in(0,1)$, and the upper bound gives tight order for an entire range of $\alpha \in(0,2)$.

Remark 2.2. A similar result can be found for an $\alpha$-stable Lévy motion in $\mathbb{R}^{N}$. In [7], Getoor established an exact expression for the mean first exit time from an $N$-ball of radius $R$. In particular, from [7, formula (A)] it follows that

$$
\mathbb{E}\left[\tau_{R}\right]=K(\alpha, N)\left(R^{2}-|\boldsymbol{x}|^{2}\right)^{\alpha / 2},
$$

where $K(\alpha, N)$ is a constant that depends on $\alpha$ and the dimension $N$, and $\boldsymbol{x}$ is the starting point of the $\alpha$-stable Lévy motion. Hence, for any starting point $\boldsymbol{x} \in \mathbb{R}^{N}$ such that $|\boldsymbol{x}|<R$, the mean first exit time scales as $\Theta\left(R^{\alpha}\right)$ for $\alpha \in(0,2)$. The work in [7] focuses directly on the mean and does not provide bounds on the distribution as in Theorem 2.1.

Proof of Corollary 2.1. Since the random variable $\tau_{R}$ takes on only positive integer values, the expectation $\mathbb{E}\left[\tau_{R}\right]$ can be obtained by

$$
\mathbb{E}\left[\tau_{R}\right]=\sum_{n=0}^{\infty} \mathbb{P}\left(\tau_{R}>n\right) \leq \sum_{n=0}^{\infty}\left(u_{R}\right)^{n}=\frac{1}{1-u_{R}},
$$

where the inequality follows from (2.1). Similarly, we have $\mathbb{E}\left[\tau_{R}\right] \geq\left(1-l_{R}\right)^{-1}$.

For Lévy flights in any dimension, as $R$ goes to $\infty, 1-u_{R}$ behaves as

$$
\lim _{R \rightarrow \infty} \frac{1-u_{R}}{R^{-\alpha}}=\lim _{R \rightarrow \infty} \frac{\mathbb{P}(|X|>R)}{R^{-\alpha}}=\frac{2 \Gamma(\alpha)}{\pi} \sin \left(\frac{\pi \alpha}{2}\right) \gamma^{\alpha} \quad(\neq 0,<\infty),
$$

where the last equality follows from (1.4). Thus, we have $\mathbb{E}\left[\tau_{R}\right]=O\left(R^{\alpha}\right)$ for $0<\alpha<2$.

Now we analyze the scaling behavior of $1-l_{R}$ for the one-dimensional Lévy flight. Note that $1-l_{R}$ reduces to

$$
1-l_{R}=\frac{1}{2 R} \int_{0}^{2 R} 1 \mathrm{~d} x-\frac{1}{2 R} \int_{0}^{2 R} F_{|X|}(x) \mathrm{d} x=\frac{1}{2 R} \int_{0}^{2 R} \mathbb{P}(|X|>x) \mathrm{d} x,
$$


and $\lim _{R \rightarrow \infty} \int_{0}^{2 R} \mathbb{P}(|X|>x) \mathrm{d} x=\mathbb{E}[|X|]$, which is infinite for $0<\alpha \leq 1$ and is finite for $1<\alpha<2$. Suppose that $0<\alpha<1$. Then the behavior of $1-l_{R}$ as $R$ goes to $\infty$ is

$$
\begin{aligned}
\lim _{R \rightarrow \infty} \frac{1-l_{R}}{R^{-\alpha}} & =\lim _{R \rightarrow \infty} \frac{1}{2 R^{1-\alpha}} \int_{0}^{2 R} \mathbb{P}(|X|>x) \mathrm{d} x \\
& =\lim _{R \rightarrow \infty} \frac{\mathbb{P}(|X|>2 R)}{(1-\alpha) R^{-\alpha}} \\
& =\frac{2 \Gamma(\alpha)}{(1-\alpha) \pi} \sin \left(\frac{\pi \alpha}{2}\right)\left(\frac{\gamma}{2}\right)^{\alpha} \quad(\neq 0,<\infty) .
\end{aligned}
$$

Here, L'Hópital's rule is used in the second equality since both the numerator and the denominator go to $\infty$. Therefore, we have $\mathbb{E}\left[\tau_{R}\right]=\Omega\left(R^{\alpha}\right)$ for $0<\alpha<1$. Now suppose that $\alpha=1$. Then, similarly to the previous case, we have

$$
\begin{aligned}
\lim _{R \rightarrow \infty} \frac{1-l_{R}}{R^{-1} \log R} & =\lim _{R \rightarrow \infty} \frac{1}{2 \log R} \int_{0}^{2 R} \mathbb{P}(|X|>x) \mathrm{d} x \\
& =\lim _{R \rightarrow \infty} \frac{\mathbb{P}(|X|>2 R)}{R^{-1}} \\
& =\frac{\gamma}{\pi} \quad(\neq 0,<\infty) .
\end{aligned}
$$

Therefore, we have $\mathbb{E}\left[\tau_{R}\right]=\Omega(R / \log R)$ for $\alpha=1$. Finally, suppose that $1<\alpha<2$. Then, similarly to the previous case again, we have

$$
\lim _{R \rightarrow \infty} \frac{1-l_{R}}{R^{-1}}=\lim _{R \rightarrow \infty} \frac{1}{2} \int_{0}^{2 R} \mathbb{P}(|X|>x) \mathrm{d} x=\frac{1}{2} \mathbb{E}[|X|] \quad(\neq 0,<\infty) .
$$

Therefore, we have $\mathbb{E}\left[\tau_{R}\right]=\Omega(R)$ for $1<\alpha<2$. The same result follows for the $N$-dimensional $(N \geq 2)$ Lévy flight by changing the upper boundary of the integral from $2 R$ to $R$. This completes the proof of Corollary 2.1 .

Finally, we establish the dependence of the first exit time on the scale parameter $\gamma$ of the $\alpha$-stable distribution. Proposition 2.1 shows that the order-wise asymptotic behavior of the first exit time of the one-dimensional Lévy flight is determined by the stability index $\alpha$. Hence, it suffices to consider step-length distributions with unit-scale parameter $\gamma=1$ when characterizing the asymptotics of the first exit time $\tau_{R}$ as the radius $R$ increases.

Proposition 2.1. Let $\tau_{(R, \alpha, \gamma)}$ be the first exit time of a Lévy flight with step-length parameters $\alpha, \gamma$ and ball of radius $R$. Then for the one-dimensional Lévy flight, we have

$$
\tau_{(R, \alpha, \gamma)} \stackrel{\mathrm{D}}{=} \tau_{(R / \gamma, \alpha, 1)}
$$

where '를 'denotes equal in distribution.

Proof. In this proof we add the subscript $(\alpha, \gamma)$ to the random variables $X$ and $Y_{n}$ as $X_{(\alpha, \gamma)}$ and $Y_{(n, \alpha, \gamma)}$ in order to explicitly denote the associated step-length parameters. Note that the characteristic functions of $\gamma X_{(\alpha, 1)}$ and $X_{(\alpha, \gamma)}$ are identical, i.e.

$$
\varphi_{\gamma X_{(\alpha, 1)}}(t)=\mathbb{E}\left[\mathrm{e}^{\mathrm{i} t \gamma X_{(\alpha, 1)}}\right]=\mathbb{E}\left[\mathrm{e}^{\mathrm{i}(\gamma t) X_{(\alpha, 1)}}\right]=\varphi_{X_{(\alpha, 1)}}(\gamma t)=\exp \left(-|\gamma t|^{\alpha}\right)=\varphi_{X_{(\alpha, \gamma)}}(t),
$$


where the last two equalities follow from (1.2). Since the characteristic function uniquely determines the distribution of a random variable, we have $\gamma X_{(\alpha, 1)} \stackrel{\mathrm{D}}{=} X_{(\alpha, \gamma)}$. In addition, it is known that $Y_{(n, \alpha, \gamma)} \stackrel{\mathrm{D}}{=} n^{1 / \alpha} X_{(\alpha, \gamma)}$ for $n \in \mathbb{N}[13]$. Hence, we obtain

$$
Y_{(n, \alpha, \gamma)} \stackrel{\mathrm{D}}{=} n^{1 / \alpha}\left(\gamma X_{(\alpha, 1)}\right)=\gamma\left(n^{1 / \alpha} X_{(\alpha, 1)}\right) \stackrel{\mathrm{D}}{=} \gamma Y_{(n, \alpha, 1)},
$$

i.e. $Y_{(n, \alpha, \gamma)} \stackrel{\mathrm{D}}{=} \gamma Y_{(n, \alpha, 1)}$. Therefore, for each $n \in \mathbb{N}$, we have

$$
\begin{aligned}
\mathbb{P}\left(\tau_{(R, \alpha, \gamma)}>n\right) & =\mathbb{P}\left(\left|Y_{(k, \alpha, \gamma)}\right| \leq R, k=1,2, \ldots, n\right) \\
& =\mathbb{P}\left(\gamma\left|Y_{(k, \alpha, 1)}\right| \leq R, k=1,2, \ldots, n\right) \\
& =\mathbb{P}\left(\tau_{(R / \gamma, \alpha, 1)}>n\right) .
\end{aligned}
$$

Since (2.23) holds for any $n \in \mathbb{N}$, we have $\tau_{(R, \alpha, \gamma)} \stackrel{\mathrm{D}}{=} \tau_{(R / \gamma, \alpha, 1)}$.

\section{Appendix A. Proof of Lemma 2.1}

The $\operatorname{CDF} F_{X}(\cdot)$ is infinitely differentiable on its domain $(-\infty, \infty)$, and the PDF $f_{X}(\cdot)$ satisfies $f_{X}\left(\left|x_{l}\right|\right) \geq f_{X}\left(\left|x_{u}\right|\right)$ for $\left|x_{l}\right| \leq\left|x_{u}\right|$ [6]. Since $F_{|X|}(x)=2 F_{X}(x)-1$ and $f_{|X|}(x)=$ $2 f_{X}(x)$ for $x \geq 0$, the result follows.

\section{Appendix B. Proof of Lemma 2.2}

We provide the proof for the $N$-dimensional $(N \geq 2)$ case by induction on $n$. The proof for the one-dimensional case follows from similar but simpler arguments.

Suppose $n=1$. Since $\left|Y_{1}\right|=\left|X_{1}\right|$ and $\left\{\tau_{R}>1\right\}=\left\{\left|X_{1}\right| \leq R\right\}$, we have

$$
f_{\left|Y_{1}\right|}\left(y \mid \tau_{R}>1\right)=f_{\left|X_{1}\right|}\left(y|| X_{1} \mid \leq R\right)=c_{1} f_{|X|}(y),
$$

where $c_{1}:=(\mathbb{P}(|X| \leq R))^{-1}$. Hence, by Lemma 2.1 , the conditional density function $f_{\left|Y_{1}\right|}\left(\cdot \mid \tau_{R}>1\right)$ is continuous, and satisfies, for $0 \leq y_{l} \leq y_{u} \leq R$,

$$
f_{\left|Y_{1}\right|}\left(y_{l} \mid \tau_{R}>1\right)=c_{1} f_{|X|}\left(y_{l}\right) \geq c_{1} f_{|X|}\left(y_{u}\right)=f_{\left|Y_{1}\right|}\left(y_{u} \mid \tau_{R}>1\right) .
$$

This proves Lemma 2.2 for $n=1$.

Suppose $n \in \mathbb{N}$. Assume that

(A1) $f_{\left|Y_{n}\right|}\left(y \mid \tau_{R}>n\right)$ exists for $0 \leq y \leq R$;

(A2) $f_{\left|Y_{n}\right|}\left(y \mid \tau_{R}>n\right)$ is continuous over its domain $[0, R]$;

(A3) for $0 \leq y_{l} \leq y_{u} \leq R, f_{\left|Y_{n}\right|}\left(y_{l} \mid \tau_{R}>n\right) \geq f_{\left|Y_{n}\right|}\left(y_{u} \mid \tau_{R}>n\right)$.

Under these assumptions, we will prove that Lemma 2.2 holds for $n+1$. We first show the existence of the conditional density function $f_{\left|Y_{n+1}\right|}\left(y \mid \tau_{R}>n+1\right)$ for $0 \leq y \leq R$. Let $B_{R}=\left\{\boldsymbol{x} \in \mathbb{R}^{N}:|\boldsymbol{x}| \leq R\right\}$. For a vector $\boldsymbol{x} \in \mathbb{R}^{N}$, let $x_{i}$ denote the $i$ th component of $\boldsymbol{x}$. For any two vectors $\boldsymbol{x}, \boldsymbol{y} \in \mathbb{R}^{N}$, we write $\boldsymbol{x} \leq \boldsymbol{y}$ to indicate $x_{i} \leq y_{i}$ for $i=1, \ldots, N$. Then, for $\boldsymbol{y} \in \mathscr{B}_{R}$, we have

$$
\begin{aligned}
\mathbb{P}\left(Y_{n+1} \leq \boldsymbol{y} \mid \tau_{R}>n+1\right) & =\frac{1}{\mathbb{P}\left(\tau_{R}>n+1\right)} \mathbb{P}\left(Y_{n+1} \leq \boldsymbol{y}, \tau_{R}>n+1\right) \\
& =\frac{1}{\mathbb{P}\left(\tau_{R}>n+1\right)} \mathbb{P}\left(Y_{n+1} \in \delta(\boldsymbol{y}), \tau_{R}>n\right) \\
& =c_{n+1} \mathbb{P}\left(Y_{n+1} \in \delta(\boldsymbol{y}) \mid \tau_{R}>n\right)
\end{aligned}
$$


where $\delta(\boldsymbol{y}):=\left\{\boldsymbol{x} \in \mathscr{B}_{R}: \boldsymbol{x} \leq \boldsymbol{y}\right\}$ and $c_{n+1}:=\mathbb{P}\left(\tau_{R}>n\right) / \mathbb{P}\left(\tau_{R}>n+1\right)$. Here, the second equality is obtained in a similar manner as in (2.10). Based on assumption (A1), we can write the probability $\mathbb{P}\left(Y_{n+1} \in \delta(\boldsymbol{y}) \mid \tau_{R}>n\right)$ by conditioning on $Y_{n}$ as

$$
\begin{aligned}
\mathbb{P}\left(Y_{n+1} \in \delta(\boldsymbol{y}) \mid \tau_{R}>n\right) & =\int_{\mathcal{B}_{R}} \mathbb{P}\left(Y_{n+1} \in \delta(\boldsymbol{y}) \mid Y_{n}=\boldsymbol{x}, \tau_{R}>n\right) f_{Y_{n}}\left(\boldsymbol{x} \mid \tau_{R}>n\right) \mathrm{d} \boldsymbol{x} \\
& =\int_{\mathcal{B}_{R}} \mathbb{P}\left(Y_{n+1} \in \delta(\boldsymbol{y}) \mid Y_{n}=\boldsymbol{x}\right) f_{Y_{n}}\left(\boldsymbol{x} \mid \tau_{R}>n\right) \mathrm{d} \boldsymbol{x} \\
& =\int_{\mathcal{B}_{R}} \int_{\delta(\boldsymbol{y})} \frac{1}{2 \pi^{N-1}} f_{|X|}(|\boldsymbol{x}-\boldsymbol{s}|) \mathrm{d} \boldsymbol{s} f_{Y_{n}}\left(\boldsymbol{x} \mid \tau_{R}>n\right) \mathrm{d} \boldsymbol{x} \\
& =\frac{1}{2 \pi^{N-1}} \int_{\delta(\boldsymbol{y})} \int_{\mathcal{B}_{R}} f_{|X|}(|\boldsymbol{x}-\boldsymbol{s}|) f_{Y_{n}}\left(\boldsymbol{x} \mid \tau_{R}>n\right) \mathrm{d} \boldsymbol{x} \mathrm{d} \boldsymbol{s} \\
& =\frac{1}{2 \pi^{N-1}} \int_{\delta(y)} \mathbb{E}\left[f_{|X|}\left(\left|Y_{n}-\boldsymbol{s}\right|\right) \mid \tau_{R}>n\right] \mathrm{d} \boldsymbol{s},
\end{aligned}
$$

where the second equality is obtained by the Markov nature of $\left\{Y_{n}\right\}_{n \in \mathbb{N}}$, and Tonelli's theorem is applied in the fourth equality given the nonnegativity of the density functions $f_{|X|}(\cdot)$ and $f_{Y_{n}}\left(\cdot \mid \tau_{R}>n\right)$. To simplify notation, we define

$$
H(s)=\frac{1}{2 \pi^{N-1}} \mathbb{E}\left[f_{|X|}\left(\left|Y_{n}-\boldsymbol{s}\right|\right) \mid \tau_{R}>n\right], \quad s \in \delta(\boldsymbol{y}) .
$$

Also, in order to differentiate (B.2) with respect to $y_{1}, \ldots, y_{N}$ sequentially, we introduce

$$
\begin{gathered}
\mathcal{V}(\boldsymbol{a})=\left\{\boldsymbol{x} \in \mathbb{R}^{k}: \boldsymbol{x} \leq \boldsymbol{a}\right\}, \quad \boldsymbol{a} \in \mathbb{R}^{k}, \\
\mathcal{B}(\boldsymbol{b})=\left\{\boldsymbol{x} \in \mathbb{R}^{k}:|\boldsymbol{x}| \leq \sqrt{R^{2}-|\boldsymbol{b}|^{2}}\right\}, \quad \boldsymbol{b} \in \mathbb{R}^{N-k},
\end{gathered}
$$

where $k=1, \ldots, N-1$. Then, we have

$$
\frac{\partial}{\partial y_{1}} \mathbb{P}\left(Y_{n+1} \in \delta(\boldsymbol{y}) \mid \tau_{R}>n\right)=\int_{\mathcal{B}\left(y_{1}\right) \cap \mathcal{V}\left(y_{2}, \ldots, y_{N}\right)} H\left(y_{1}, s_{2}, \ldots, s_{N}\right) \mathrm{d}\left(s_{2}, \ldots, s_{N}\right) .
$$

In deriving (B.4), we first decompose $\delta(\boldsymbol{y})$ as

$$
\begin{aligned}
\&(\boldsymbol{y})= & \left\{\boldsymbol{s} \in \mathbb{R}^{N}:\left|s_{1}\right| \leq R,\left|\left(s_{2}, \ldots, s_{N}\right)\right| \leq \sqrt{R^{2}-\left(s_{1}\right)^{2}}\right\} \\
& \bigcap\left\{\boldsymbol{s} \in \mathbb{R}^{N}: s_{1} \leq y_{1},\left(s_{2}, \ldots, s_{N}\right) \leq\left(y_{2}, \ldots, y_{N}\right)\right\} \\
= & \left\{\boldsymbol{s} \in \mathbb{R}^{N}: s_{1} \in\left[-R, y_{1}\right],\left(s_{2}, \ldots, s_{N}\right) \in \mathcal{B}\left(s_{1}\right) \cap \mathcal{V}\left(y_{2}, \ldots, y_{N}\right)\right\} .
\end{aligned}
$$

Next, we combine (B.2), (B.3), and (B.5) to obtain

$$
\mathbb{P}\left(Y_{n+1} \in \delta(\boldsymbol{y}) \mid \tau_{R}>n\right)=\int_{-R}^{y_{1}} \int_{\mathcal{B}\left(s_{1}\right) \cap \mathcal{V}\left(y_{2}, \ldots, y_{N}\right)} H(s) \mathrm{d}\left(s_{2}, \ldots, s_{N}\right) \mathrm{d} s_{1} .
$$

Based on the continuity assumption (A2), we can apply the fundamental theorem of calculus to find the derivative of (B.6) with respect to $y_{1}$, which results in (B.4).

Using a similar approach as above, we have

$$
\begin{aligned}
& \frac{\partial^{2}}{\partial\left(y_{2}, y_{1}\right)} \mathbb{P}\left(Y_{n+1} \in \delta(\boldsymbol{y}) \mid \tau_{R}>n\right) \\
& \quad=\frac{\partial}{\partial y_{2}} \int_{\mathcal{B}\left(y_{1}\right) \cap \mathcal{V}\left(y_{2}, \ldots, y_{N}\right)} H\left(y_{1}, s_{2}, \ldots, s_{N}\right) \mathrm{d}\left(s_{2}, \ldots, s_{N}\right)
\end{aligned}
$$




$$
\begin{aligned}
& =\frac{\partial}{\partial y_{2}} \int_{-\sqrt{R^{2}-y_{1}^{2}}}^{y_{2}} \int_{\mathcal{B}\left(y_{1}, s_{2}\right) \cap \mathcal{V}\left(y_{3}, \ldots, y_{N}\right)} H\left(y_{1}, s_{2}, \ldots, s_{N}\right) \mathrm{d}\left(s_{3}, \ldots, s_{N}\right) \mathrm{d} s_{2} \\
& =\int_{\mathcal{B}\left(y_{1}, y_{2}\right) \cap \mathcal{V}\left(y_{3}, \ldots, y_{N}\right)} H\left(y_{1}, y_{2}, s_{3}, \ldots, s_{N}\right) \mathrm{d}\left(s_{3}, \ldots, s_{N}\right) .
\end{aligned}
$$

Continuing this process, we finally obtain

$$
\frac{\partial^{N}}{\partial\left(y_{N}, \ldots, y_{1}\right)} \mathbb{P}\left(Y_{n+1} \in \&(\boldsymbol{y}) \mid \tau_{R}>n\right)=H(\boldsymbol{y}) .
$$

Also, by the isotropy of the Lévy flight, we have

$$
\frac{\partial^{N}}{\partial\left(y_{i_{1}}, y_{i_{2}}, \ldots, y_{i_{N}}\right)} \mathbb{P}\left(Y_{n+1} \in \delta(\boldsymbol{y}) \mid \tau_{R}>n\right)=H(\boldsymbol{y})
$$

for any permutation $\left(i_{1}, i_{2}, \ldots, i_{N}\right)$ of $(1,2, \ldots, N)$. Hence, for a point $\boldsymbol{y} \in \mathscr{B}_{R}$, the density function $f_{Y_{n+1}}\left(\boldsymbol{y} \mid \tau_{R}>n+1\right)$ exists and is obtained from (B.1) and (B.7) by

$$
f_{Y_{n+1}}\left(\boldsymbol{y} \mid \tau_{R}>n+1\right)=c_{n+1} H(\boldsymbol{y})=\frac{c_{n+1}}{2 \pi^{N-1}} \mathbb{E}\left[f_{|X|}\left(\left|Y_{n}-\boldsymbol{y}\right|\right) \mid \tau_{R}>n\right] .
$$

By the isotropy of the Lévy flight again, we have, for $0 \leq y \leq R$,

$$
\begin{aligned}
f_{\left|Y_{n+1}\right|}\left(y \mid \tau_{R}>n+1\right) & =2 \pi^{N-1} f_{Y_{n+1}}\left(y \boldsymbol{e}_{1} \mid \tau_{R}>n+1\right) \\
& =c_{n+1} \mathbb{E}\left[f_{|X|}\left(\left|Y_{n}-y \boldsymbol{e}_{1}\right|\right) \mid \tau_{R}>n\right],
\end{aligned}
$$

where $\boldsymbol{e}_{1}$ denotes the first standard basis vector in $\mathbb{R}^{N}$, i.e. $\boldsymbol{e}_{1}=(1,0, \ldots, 0) \in \mathbb{R}^{N}$. This proves the existence of the density function $f_{\left|Y_{n+1}\right|}\left(\cdot \mid \tau_{R}>n+1\right)$.

We next prove the continuity of $f_{\left|Y_{n+1}\right|}\left(\cdot \mid \tau_{R}>n+1\right)$. To this end, we show that $f_{\left|Y_{n+1}\right|}\left(\cdot \mid \tau_{R}>n+1\right)$ is differentiable, and its derivative is given by

$$
\frac{\mathrm{d}}{\mathrm{d} y} f_{\left|Y_{n+1}\right|}\left(y \mid \tau_{R}>n+1\right)=c_{n+1} \int_{\mathcal{B}_{R}} \frac{\left(y-s_{1}\right) f_{|X|}^{\prime}\left(\left|\boldsymbol{s}-y \boldsymbol{e}_{1}\right|\right) f_{Y_{n}}\left(\boldsymbol{s} \mid \tau_{R}>n\right)}{\left|\boldsymbol{s}-y \boldsymbol{e}_{1}\right|} \mathrm{d} \boldsymbol{s},
$$

where $f_{|X|}^{\prime}(x):=(\mathrm{d} / \mathrm{d} x)\left(f_{|X|}(x)\right)$. From (B.8), we have

$$
\begin{aligned}
& \frac{\mathrm{d}}{\mathrm{d} y} f_{\left|Y_{n+1}\right|}\left(y \mid \tau_{R}>n+1\right) \\
& \quad=\lim _{\varepsilon \rightarrow 0} \frac{f_{\left|Y_{n+1}\right|}\left(y+\varepsilon \mid \tau_{R}>n+1\right)-f_{\left|Y_{n+1}\right|}\left(y \mid \tau_{R}>n+1\right)}{\varepsilon} \\
& \quad=c_{n+1} \lim _{\varepsilon \rightarrow 0} \int_{\mathcal{B}_{R}} \frac{f_{|X|}\left(\left|s-(y+\varepsilon) \boldsymbol{e}_{1}\right|\right)-f_{|X|}\left(\left|\boldsymbol{s}-y \boldsymbol{e}_{1}\right|\right)}{\varepsilon} f_{Y_{n}}\left(\boldsymbol{s} \mid \tau_{R}>n\right) \mathrm{d} \boldsymbol{s} .
\end{aligned}
$$

In this proof we assume that $\varepsilon$ in (B.10) is positive, but we can prove the case $\varepsilon<0$ similarly. For each fixed point $s \in \mathscr{B}_{R}$, the density $f_{|X|}\left(\left|\boldsymbol{s}-y \boldsymbol{e}_{1}\right|\right)$ is an infinitely differentiable function of $y$ by Lemma 2.1, and, thus, by the mean value theorem, there exists $\bar{y} \in(y, y+\varepsilon)$ such that

$$
\frac{f_{|X|}\left(\left|s-(y+\varepsilon) \boldsymbol{e}_{1}\right|\right)-f_{|X|}\left(\left|s-y \boldsymbol{e}_{1}\right|\right)}{\varepsilon}=f_{|X|}^{\prime}\left(\left|s-\bar{y} \boldsymbol{e}_{1}\right|\right) \frac{\bar{y}-s_{1}}{\left|s-\bar{y} \boldsymbol{e}_{1}\right|} .
$$


The term on the right-hand side of (B.11) is bounded by

$$
\left|f_{|X|}^{\prime}\left(\left|\boldsymbol{s}-\bar{y} \boldsymbol{e}_{1}\right|\right) \frac{\bar{y}-s_{1}}{\left|\boldsymbol{s}-\bar{y} \boldsymbol{e}_{1}\right|}\right| \leq \sup _{0 \leq x \leq 2 R}\left|f_{|X|}^{\prime}(x)\right|\left|\frac{\bar{y}-s_{1}}{\left|\left(s_{1}-\bar{y}\right) \boldsymbol{e}_{1}\right|}\right|=M,
$$

where $M:=\sup _{0 \leq x \leq 2 R}\left|f_{|X|}^{\prime}(x)\right|$ is finite since $f_{|X|}^{\prime}(\cdot)$ is continuous by Lemma 2.1. Hence, the first term inside the integral in (B.10) is bounded for any $s \in \mathcal{B}_{R}, y \in[0, R]$, and $\varepsilon>0$ by

$$
\left|\frac{f_{|X|}\left(\left|s-(y+\varepsilon) \boldsymbol{e}_{1}\right|\right)-f_{|X|}\left(\left|s-y \boldsymbol{e}_{1}\right|\right)}{\varepsilon}\right| \leq M .
$$

The second term inside the integral in (B.10) is bounded for any $s \in \mathcal{B}_{R}$ by

$$
f_{Y_{n}}\left(s \mid \tau_{R}>n\right)=\frac{1}{2 \pi^{N-1}} f_{\left|Y_{n}\right|}\left(|s| \mid \tau_{R}>n\right) \leq \frac{1}{2 \pi^{N-1}} f_{\left|Y_{n}\right|}\left(0 \mid \tau_{R}>n\right),
$$

where the inequality follows from assumption (A3). Given (B.12) and (B.13), we can apply the dominated convergence theorem to (B.10) to obtain

$$
\begin{aligned}
\lim _{\varepsilon \rightarrow 0} & \frac{f_{\left|Y_{n+1}\right|}\left(y+\varepsilon \mid \tau_{R}>n+1\right)-f_{\left|Y_{n+1}\right|}\left(y \mid \tau_{R}>n+1\right)}{\varepsilon} \\
& =c_{n+1} \int_{\mathcal{B}_{R}} \lim _{\varepsilon \rightarrow 0} \frac{f_{|X|}\left(\left|\boldsymbol{s}-(y+\varepsilon) \boldsymbol{e}_{1}\right|\right)-f_{|X|}\left(\left|\boldsymbol{s}-y \boldsymbol{e}_{1}\right|\right)}{\varepsilon} f_{Y_{n}}\left(\boldsymbol{s} \mid \tau_{R}>n\right) \mathrm{d} \boldsymbol{s} \\
& =c_{n+1} \int_{\mathcal{B}_{R}} f_{|X|}^{\prime}\left(\left|\boldsymbol{s}-y \boldsymbol{e}_{1}\right|\right) \frac{y-s_{1}}{\left|\boldsymbol{s}-y \boldsymbol{e}_{1}\right|} f_{Y_{n}}\left(\boldsymbol{s} \mid \tau_{R}>n\right) \mathrm{d} \boldsymbol{s},
\end{aligned}
$$

where the second equality follows from (B.11). This proves (B.9).

Finally, we prove the monotonicity of $f_{\left|Y_{n+1}\right|}\left(\cdot \mid \tau_{R}>n+1\right)$. Fix $y_{l}$ and $y_{u}$ such that $0 \leq y_{l} \leq y_{u} \leq R$. From (B.8), we have

$$
\begin{aligned}
& f_{\left|Y_{n+1}\right|}\left(y_{l} \mid \tau_{R}>n+1\right)-f_{\left|Y_{n+1}\right|}\left(y_{u} \mid \tau_{R}>n+1\right) \\
& \quad=c_{n+1} \mathbb{E}\left[f_{|X|}\left(\left|Y_{n}-y_{l} \boldsymbol{e}_{1}\right|\right)-f_{|X|}\left(\left|Y_{n}-y_{u} \boldsymbol{e}_{1}\right|\right) \mid \tau_{R}>n\right] \\
& \quad=c_{n+1} \int_{\mathcal{B}_{R}} J(\boldsymbol{s}) f_{Y_{n}}\left(\boldsymbol{s} \mid \tau_{R}>n\right) \mathrm{d} \boldsymbol{s},
\end{aligned}
$$

where

$$
J(s):=f_{|X|}\left(\left|s-y_{l} \boldsymbol{e}_{1}\right|\right)-f_{|X|}\left(\left|s-y_{u} \boldsymbol{e}_{1}\right|\right) .
$$

Given $y_{l}$ and $y_{u}$, we partition the ball $\mathscr{B}_{R}$ into three disjoint regions $\mathscr{D}_{1}, \mathscr{D}_{2}$, and $\mathscr{D}_{3}$ as

$$
\begin{gathered}
\mathscr{D}_{1}:=\left\{s \in \mathcal{B}_{R}: s_{1} \geq \frac{y_{l}+y_{u}}{2}\right\}, \quad \mathscr{D}_{2}:=\left\{\left(y_{l}+y_{u}\right) \boldsymbol{e}_{1}-\boldsymbol{s} \in \mathscr{B}_{R}: s \in \mathscr{D}_{1}\right\}, \\
\mathscr{D}_{3}:=\mathscr{B}_{R} \backslash\left(\mathscr{D}_{1} \cup \mathscr{D}_{2}\right) ;
\end{gathered}
$$

see Figure 1 for a depiction of $\mathscr{D}_{1}, \mathscr{D}_{2}$, and $\mathscr{D}_{3}$ in $\mathbb{R}^{2}$. Note that $\mathscr{D}_{2}$ is the mirror image of $\mathscr{D}_{1}$ with respect to the point $\left(\left(y_{l}+y_{u}\right) / 2\right) \boldsymbol{e}_{1}$, and $\mathscr{D}_{2} \cup \mathscr{D}_{3}=\left\{\boldsymbol{s} \in \mathscr{B}_{R}: s_{1} \leq\left(y_{l}+y_{u}\right) / 2\right\}$. Also, note that $\left|\boldsymbol{s}-y_{l} \boldsymbol{e}_{1}\right| \leq\left|\boldsymbol{s}-y_{u} \boldsymbol{e}_{1}\right|$ if and only if $s_{1} \leq\left(y_{l}+y_{u}\right) / 2$. Hence, by Lemma 2.1, $J(s) \leq 0$ for $s \in \mathscr{D}_{1}$ and $J(s) \geq 0$ for $s \in \mathscr{D}_{2} \cup \mathscr{D}_{3}$, which yields

$$
\begin{gathered}
\int_{\mathscr{D}_{1}} J(s) f_{Y_{n}}\left(s \mid \tau_{R}>n\right) \mathrm{d} s \leq 0, \quad \int_{D_{2}} J(s) f_{Y_{n}}\left(s \mid \tau_{R}>n\right) \mathrm{d} s \geq 0 \\
\int_{\mathscr{D}_{3}} J(s) f_{Y_{n}}\left(s \mid \tau_{R}>n\right) \mathrm{d} s \geq 0 .
\end{gathered}
$$




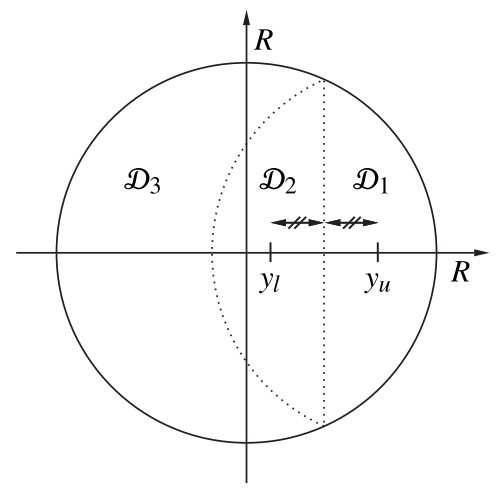

FIGURE 1: Geometry in the proof of Lemma 2.2

We will compare the first two integrals in (B). For each point $s \in \mathcal{D}_{1}$, we define

$$
\hat{\boldsymbol{s}}=\left(y_{l}+y_{u}\right) \boldsymbol{e}_{1}-\boldsymbol{s}
$$

to simplify the notation. Then, we have $\left|\boldsymbol{s}-y_{l} \boldsymbol{e}_{1}\right|=\left|\hat{\boldsymbol{s}}-y_{u} \boldsymbol{e}_{1}\right|$ and $\left|\boldsymbol{s}-y_{u} \boldsymbol{e}_{1}\right|=\left|\hat{\boldsymbol{s}}-y_{l} \boldsymbol{e}_{1}\right|$. Accordingly, we have

$$
J(s)=-J(\hat{s}) \leq 0 \quad \text { for } s \in D_{1}
$$

In addition, since $|\boldsymbol{s}| \geq|\hat{\boldsymbol{s}}|$ for $\boldsymbol{s} \in \mathcal{D}_{1}$, we have $f_{\left|Y_{n}\right|}\left(|\boldsymbol{s}| \mid \tau_{R}>n\right) \leq f_{\left|Y_{n}\right|}\left(|\hat{\boldsymbol{s}}| \mid \tau_{R}>n\right)$ by assumption (A3). Thus, we have

$$
f_{Y_{n}}\left(\boldsymbol{s} \mid \tau_{R}>n\right) \leq f_{Y_{n}}\left(\hat{\boldsymbol{s}} \mid \tau_{R}>n\right) \text { for } \boldsymbol{s} \in \mathscr{D}_{1}
$$

From (B.17) and (B.18), we have

$$
\begin{aligned}
\int_{\mathbb{D}_{1}} J(\boldsymbol{s}) f_{Y_{n}}\left(\boldsymbol{s} \mid \tau_{R}>n\right) \mathrm{d} \boldsymbol{s} & \geq-\int_{D_{1}} J(\hat{\boldsymbol{s}}) f_{Y_{n}}\left(\hat{\boldsymbol{s}} \mid \tau_{R}>n\right) \mathrm{d} \boldsymbol{s} \\
& =-\int_{D_{1}} J\left(\left(y_{l}+y_{u}\right) \boldsymbol{e}_{1}-\boldsymbol{s}\right) f_{Y_{n}}\left(\left(y_{l}+y_{u}\right) \boldsymbol{e}_{1}-\boldsymbol{s} \mid \tau_{R}>n\right) \mathrm{d} \boldsymbol{s} \\
& =-\int_{D_{2}} J(\tilde{\boldsymbol{s}}) f_{Y_{n}}\left(\tilde{\boldsymbol{s}} \mid \tau_{R}>n\right) \mathrm{d} \tilde{\boldsymbol{s}}
\end{aligned}
$$

where the second equality is obtained by the definition of $\mathscr{D}_{2}$. Hence, we have

$$
\int_{D_{1} \cup D_{2}} J(s) f_{Y_{n}}\left(s \mid \tau_{R}>n\right) \mathrm{d} \boldsymbol{s} \geq 0 .
$$

Combining (B.14), (B), and (B.19) shows the monotonicity of $f_{\left|Y_{n+1}\right|}\left(\cdot \mid \tau_{R}>n+1\right)$. This completes the proof of Lemma 2.2. 


\section{Appendix C. Proof of Lemma 2.3}

We prove the one-dimensional case first. Starting with the definition of $G(\cdot)$, we have for any $y \in[0, R]$ the following:

$$
\begin{aligned}
G(y) & :=\mathbb{P}\left(\left|Y_{n+1}\right| \leq R|| Y_{n} \mid=y\right) \\
& =\mathbb{P}\left(\left|Y_{n+1}\right| \leq R \mid Y_{n}=y\right) \\
& =\frac{1}{2} \mathbb{P}\left(\left|X_{n+1}\right| \leq R-y\right)+\frac{1}{2} \mathbb{P}\left(\left|X_{n+1}\right| \leq R+y\right) \\
& =\frac{1}{2} F_{|X|}(R-y)+\frac{1}{2} F_{|X|}(R+y) .
\end{aligned}
$$

Here, the second equality follows from the symmetry of the Lévy flight $\left\{Y_{n}\right\}_{n \in \mathbb{N}}$ in $\mathbb{R}$. Since $F_{|X|}(\cdot)$ is differentiable by Lemma $2.1, G(\cdot)$ is also differentiable, and its derivative satisfies

$$
\frac{\mathrm{d}}{\mathrm{d} y} G(y)=-\frac{1}{2} f_{|X|}(R-y)+\frac{1}{2} f_{|X|}(R+y) \leq 0
$$

where the inequality follows from Lemma 2.1. Hence, we have $G\left(y_{l}\right) \geq G\left(y_{u}\right)$ for any $y_{l}$ and $y_{u}$ with $0 \leq y_{l} \leq y_{u} \leq R$. This proves Lemma 2.3 for the one-dimensional case.

We now prove the $N$-dimensional $(N \geq 2)$ case. By isotropy, $G(y)$ is given by

$$
G(y):=\mathbb{P}\left(\left|Y_{n+1}\right| \leq R|| Y_{n} \mid=y\right)=\mathbb{P}\left(\left|Y_{n+1}\right| \leq R \mid Y_{n}=y \boldsymbol{e}_{1}\right) .
$$

By conditioning on $\theta_{n+1}$ in (C.1), we have

$$
\begin{aligned}
G(y) & =\int_{\Omega} \mathbb{P}\left(\left|Y_{n+1}\right| \leq R \mid Y_{n}=y \boldsymbol{e}_{1}, \theta_{n+1}=\omega\right) f_{\theta_{n+1}}\left(\boldsymbol{\omega} \mid Y_{n}=y \boldsymbol{e}_{1}\right) \mathrm{d} \boldsymbol{} \\
& =\int_{\Omega} \mathbb{P}\left(\left|Y_{n}+\right| X_{n+1}\left|\Lambda\left(\theta_{n+1}\right)\right| \leq R \mid Y_{n}=y \boldsymbol{e}_{1}, \theta_{n+1}=\omega\right) \frac{1}{2 \pi^{N-1}} \mathrm{~d} \boldsymbol{} \\
& =\frac{1}{2 \pi^{N-1}} \int_{\Omega} \mathbb{P}\left(\left|y \boldsymbol{e}_{1}+\right| X_{n+1}|\Lambda(\omega)| \leq R\right) \mathrm{d} \boldsymbol{\omega},
\end{aligned}
$$

where the last equality follows from the independence of $X_{n+1}, \theta_{n+1}$, and $Y_{n}$. By the triangle inequality, we have $\left|y \boldsymbol{e}_{1}+\right| X_{n+1}|\Lambda(\boldsymbol{\omega})| \leq\left|y \boldsymbol{e}_{1}\right|+|| X_{n+1}|\Lambda(\boldsymbol{\omega})|=y+\left|X_{n+1}\right|$, which shows that the event $\left\{y+\left|X_{n+1}\right| \leq R\right\}$ implies the event $\left\{\left|y \boldsymbol{e}_{1}+\right| X_{n+1}|\Lambda(\omega)| \leq R\right\}$. Hence, we have $\mathbb{P}\left(\left|y \boldsymbol{e}_{1}+\right| X_{n+1}|\Lambda(\omega)| \leq R\right) \geq \mathbb{P}\left(y+\left|X_{n+1}\right| \leq R\right)=F_{|X|}(R-y)$ for any $\omega \in \Omega$. Therefore, from (C.2), we have $G(y) \geq F_{|X|}(R-y)$.

To prove the monotonicity of $G(\cdot)$, we represent $G(y)$ in (C.1) as

$$
G(y)=\mathbb{P}\left(Y_{n+1} \in \mathcal{B}_{R} \mid Y_{n}=y \boldsymbol{e}_{1}\right)=\int_{\mathcal{B}_{R}} \frac{1}{2 \pi^{N-1}} f_{|X|}\left(\left|\boldsymbol{s}-y \boldsymbol{e}_{1}\right|\right) \mathrm{d} \boldsymbol{s} .
$$

Hence, for fixed $y_{l}$ and $y_{u}$, the difference $G\left(y_{l}\right)-G\left(y_{u}\right)$ is given by

$$
G\left(y_{l}\right)-G\left(y_{u}\right)=\frac{1}{2 \pi^{N-1}} \int_{\mathcal{B}_{R}}\left[f_{|X|}\left(\left|\boldsymbol{s}-y_{l} \boldsymbol{e}_{1}\right|\right)-f_{|X|}\left(\left|s-y_{u} \boldsymbol{e}_{1}\right|\right)\right] \mathrm{d} \boldsymbol{s} .
$$

Note that the integrand in (C.3) becomes $f_{|X|}\left(\left|s-y_{l} \boldsymbol{e}_{1}\right|\right)-f_{|X|}\left(\left|s-y_{u} \boldsymbol{e}_{1}\right|\right)=J(s)$, which we have defined in (B.15) in the proof of Lemma 2.2. Following the same argument from (B.15) through (B.19) but replacing $f_{Y_{n}}\left(\cdot \mid \tau_{R}>n\right)$ with 1 , we can readily obtain

$$
\int_{\mathcal{B}_{R}} J(s) \mathrm{d} s=\int_{\mathscr{D}_{1} \cup D_{2}} J(s) \mathrm{d} s+\int_{\mathscr{D}_{3}} J(s) \mathrm{d} s=\int_{\mathscr{D}_{3}} J(s) \mathrm{d} s \geq 0 .
$$

Combining (C.3) and (C.4) yields $G\left(y_{l}\right)-G\left(y_{u}\right) \geq 0$. This completes the proof. 


\section{Acknowledgements}

The authors would like to thank the anonymous referee for his/her comments on the paper, which have helped to substantially improve the quality of the paper. This research was supported in part by the Army Research Office Multidisciplinary University Research Initiative (grant no. W911NF-12-1-0385) and Basic Science Research Program through the National Research Foundation of Korea (NRF) funded by the Ministry of education (grant no. NRF2014R1A1A2057793).

\section{References}

[1] Andersen, E. Sparre (1953). On sums of symmetrically dependent random variables. Scand. Actuarial J. 1953, $123-138$.

[2] Buldyrev, S. V. et al. (2001). Average time spent by Lévy flights and walks on an interval with absorbing boundaries. Phys. Rev. E 64, 041108.

[3] Buldyrev, S. V. et al. (2001). Properties of Lévy flights on an interval with absorbing boundaries. Physica A 302, 148-161.

[4] Chen, Z.-Q., Kim, P. And Song, R. (2010). Heat kernel estimates for the Dirichlet fractional Laplacian. J. Eur. Math. Soc. 12, 1307-1329.

[5] Dybiec, B., Gudowska-Nowak, E. ANd HäNGGi, P. (2006). Lévy-Brownian motion on finite intervals: mean first passage time analysis. Phys. Rev. E 73, 046104.

[6] Gawronski, W. (1984). On the bell-shape of stable densities. Ann. Prob. 12, 230-242.

[7] Getoor, R. K. (1961). First passage times for symmetric stable processes in space. Trans. Amer. Math. Soc. 101, 75-90.

[8] Katzav, E. And AdDa-Bedia, M. (2008). The spectrum of the fractional Laplacian and first-passage-time statistics. EPL 83, 30006.

[9] Pruitt, W. E. (1981). The growth of random walks and Lévy processes. Ann. Prob. 9, 948-956.

[10] Vahabi, M., Schulz, J. H. P., Shokri, B. And Metzler, R. (2013). Area coverage of radial Lévy flights with periodic boundary conditions. Phys. Rev. E 87, 042136.

[11] Whitт, W. (2002). Stochastic-Process Limits: An Introduction to Stochastic-Process Limits and Their Application to Queues. Springer, New York.

[12] Zoia, A., Rosso, A. And Kardar, M. (2007). Fractional Laplacian in bounded domains. Phys. Rev. E 76, 021116.

[13] Zolotarev, V. M. (1986). One-Dimensional Stable Distributions. American Mathematical Society, Providence, RI. 